\title{
STOCHASTIC MODEL FOR THE REAL-TIME TRAIN RESCHEDULING
}

\author{
B. DAVYDOV ${ }^{1}$, V. CHEBOTAREV ${ }^{2}$ \& K. KABLUKOVA ${ }^{2}$ \\ ${ }^{1}$ Far Eastern State Transport University, Russia. \\ ${ }^{2}$ Computing Center, Far Eastern Branch, Russian Academy of Sciences, Russia.
}

\begin{abstract}
The article explores the problem of train rescheduling based on the actual situation. The proposed stochastic model uses specific distributions of operating times which are dependent on the current traffic conditions. The arrival time distribution is considered as a result of adjusting the train trajectory by speed control. The results of modelled arrival distributions correspond well with the experimental data received at the Russian Railways. The proposed model is used for prevention of sequence-of-trains conflicts and violations of connections. The basis of deviation prediction is two-train model of mesalevel which uses actual features of scattering of the operation times both at sites and at stations. The article also proposed a new measure of arrival delay which considers the share of satisfied passengers. Keywords: local adjustments, online rescheduling, stochastic mesa-mode, train traffic.
\end{abstract}

\section{INTRODUCTION}

Modelling the train traffic on the railway section is necessary for the optimal schedule creation. Ensuring a high level of traffic stability is the aim of constructing a tactical schedule. There is a need to use the online rescheduling when there is assumed a disrupting process. Modelling in both of these cases has significant differences. Tactical graphic takes into account the average process conditions. A stochastic model for the schedule calculation is based on fixed probability densities that characterize the operating times. Such a model very approximately describes the real traffic options because of using a large diversity of situations and averaged densities. It is necessary to include sufficiently big buffer and running time supplements in the schedule to partially eliminate this disadvantage.

Distribution of the train arrivals at a certain station is formed from the scattering of travel and stopping times. There are prevalent models that take into account only random deviations of travel times. The known models of other type suggest that adjustment of traffic is made at the stations only in order to eliminate delays. This activity is to relocate the departure moments or change the order of trains. In each individual case, use rigid rules to resolve conflicts between trains which arrive at the transfer station. Such a regulatory framework is used in the model of Berger et al. [1]. Using the simplified model and hard algorithms of conflict resolution is useful when creating a normative schedule which however is a rough approximation to reality. This assumes the absence of operative dispatching which is a fundamental drawback of the existing methods of solving long-term scheduling problems. Section 3 discusses the main points of creation of the generic stochastic model which combines the two specified approaches. 


\section{COMPRAIL}

Operative correction of train traffic can significantly improve the quality of functioning of the railway section. The main feature is to carry out an elementary cycle of modelling for that local area where there is a single disturbance. The deviation arises in some a 'cone of delays' if of moving an intense train flow. It is necessary to operatively change the trajectory of each of the trains to eliminate the spread of delays. This process is realized through the efforts of train manager and drivers. The train dispatcher implements centralized control of the entire system function, while the train drivers solve local problems on delays compensation. This all-inclusive technique is not reflected in the existing models.

The models used in the known papers have two drawbacks. One drawback is the lack of a framework that reflects a purposeful driver's operation (or of on-board unit control) for the perturbation compensation. Another disadvantage is that they ignore the following facts. The distributions that characterize partial travel times are distinct at different times of day and in different situations. Obviously, in the model which is used for online regulation, the parameters of these distributions should be established based on the emerging conditions. Section 4 discusses the impact of the specific situation at mentioned parameters.

The stochastic model which comes really close to our approach is in Büker and Seybold [2]. They basically have the same form of distribution of the train arrivals and respective waiting policies. However, they concentrated on train interactions independent of the specific operating conditions and of activities of the personnel. We will enhance their model to analyse the impact of actual factors on the distribution of arrival times.

Computer experiments show that an output distribution width increases with the expansion of the forecast horizon in case of weak control algorithm. In many cases, this modelling result does not correspond to the actual movement of trains. When using the effective adjustments which are implemented by managers and drivers, the distribution width is stabilized over time. The problem of the effect of the driver operation on the distribution of the train arrival moments is explored in Section 5. Combining techniques of forming delays on sections and stations allows you to create the generalized stochastic model of train traffic. The basis of the formal description of the motion is two-train model that is consistently applied to all pairs of train. A generalized representation of the traffic and the process of conflicts arising is a probability graph.

\section{LITERATURE REVIEW}

Many scholars study the rescheduling problem which considers the way to recover from a disturbed movement of trains. The researches were devoted to the algorithms that optimally adjust the schedule. The well-known papers $[3,4]$ provide an extensive survey of recovery models and algorithms for real-time rescheduling. Depending on the focus, the models used to solve the scheduling problem that differs by levels of infrastructure and traffic detail. A line appears as a sequence of segments between two major stations with few intermediate stations, while a network is composed of one or several junctions of lines. Macroscopic models contain least details and have a more aggregated representation of some resources (e.g. a station), while microscopic representation includes a lot more detail such as block sections. Existing models are not focused on direct description of the points of conflicts between trains.

Analyses of previous studies show an increasing interest in stochastic approaches and models that help create adequate forecasts and make optimal decisions for traffic recovery. One of the first papers considering this problem [5] proposed analytical method for the determination of delays by a convolution of the initial (inlet area) and current delays. The analysis was limited because it used the assumption of uniform distribution of secondary delays. In 
reality, this assumption is not confirmed. Most stochastic models to analyse propagation of delays focused on single-track sections or on simple junctions. Classical stochastic models for the propagation of delays had been studied intensively, most importantly in Refs [6, 7]. The papers proposed the approximation of delay distributions to reduce the computational effort. The paper [7] represents train traffic in a form of occupying an intermediate position between the macro-models and the models with very detailed description of the process, i.e. micro-models. Cumulative distribution of delays is calculated from the sequence of activities, which is defined by a stochastic event graph.

Stochastic analytical model for a traffic on a double-track line was proposed in Ref. [8]. The paper investigates delays of a fast train caught behind slower ones. The section is modelled as an infinite server. The running time distributions for each service are obtained by solving a system of linear differential equations. This model gives a good insight into delay propagation on a simple line but becomes too complicated to handle when dealing with largescale real-world networks. The approach for predicting waiting times using queuing system with a semi-Markovian kernel was presented in Ref. [9]. Description of the service process is based on an application of the theory of blocking times and minimum headway times.

A stochastic model for delay propagation and forecasts of arrival and departure events was proposed in Ref. [1]. The model includes the general train waiting policies on stations and considers discrete distributions of travel time profiles. The model is formulated with respect to a directed event graph. Another model proposed in Ref. [10] is also designed to assess the knock-on delays. The analytical probabilistic model takes into account the duration of stops, stochastic relationship between the trajectories of trains, speed fluctuations and dynamic delay propagation. A new scheme was proposed for the exact determination of parameters of the distribution model based on the maximum likelihood method.

Stochastic simplified model can be used to find an optimal allocation of the running time supplements of a train on a number of consecutive trips along the same line [11]. The result of this study is the optimal distribution of supplements between the site elements. The approach is based on the periodic event scheduling developed in Ref. [12]. An analytical method such as the max-plus algebra (MPA) is used to evaluate stability of the schedule.

Various known models describe the functions of frequency and length of random traffic deviations. Thus, the exponential distribution is used as a stochastic model of every component [7-9]. Changes of distribution are due to 'internal' reasons. Obviously, accumulation of the individual random variables leads to exponential behaviour of delays. This problem is explored in Ref. [13] and requires further study especially for the mixed flow of passenger and freight trains.

Berger et al. [1] solve the problem of train traffic rescheduling on a large network. Scattering of arrival moments is treated as a random variable generated by a spread of the departure times and the running intervals. The basis of the analysis is two-train model. This model allows to define the order of departure of the train from the initial station and distribution of the respective moments of departure. The authors use a discrete representation of distributions.

A number of studies considered a model that comprehends two train runs, each with transferring passengers at some stations [14]. The model is illustrated as a Petri net graph. The approach proposed in Ref. [2] uses a probabilistic operational graph, which considers conflicts within transfer operation. The interlinking conflicts are mapped in the model by manipulating the time distributions of the elementary activities. The paper uses two-train mesoscopic model and the FIFO rule for conflict resolution. Use of both conditional and unconditional 


\section{COMPRAIL}

convolution is specifically made. Various types of delay are modelled by means of cumulative distribution function. Described model is logically incomplete. The authors consider only the station and crossing as the conflict points. To complete the model, you must use a resolution of the sequence-of-trains conflicts at a section.

A significant number of studies are devoted to the development of criteria for determining the optimal solutions for train traffic management. Top-level rail experts created a set of railway performance indicators that measure efficiency from the perspectives of the government, passenger/client, infrastructure manager and train operating company [15]. A very common measure to quantify the service quality is to calculate the punctuality at different nodes in the system [12]. Using only the punctuality criterion has some drawbacks. It does not take into account the number of passengers affected by delays nor does it cover the case where passengers are missing connecting trains. The new approach overcomes the above drawbacks, as it accounts for passenger delays (personal passenger time is measured in minutes) instead of only delays [10]. However, the total duration of delays weakly reflects satisfaction of the passengers. Obviously, there is a certain threshold mechanism, where the allowable time limits depend on the non-nervous traveller sentiment.

Previous studies have used the distributions of typical operations (running, stopping) in the probabilistic model that are unchanged in time and do not depend on the coordinates of a particular path element. It is required to take into account the circumstances in a particular local problem, individual distributions of which depend on the influencing factors. It is also necessary to model the impact of the online system adjustment at the quality of train traffic. Ways to solve these problems are described in the following sections.

\section{MESOSCOPIC STOCHASTIC MODELLING OF TRAIN TRAFFIC}

Two types of deterministic models are used in the analysis of intensive train traffic. The micro-model reflects the train running with a readability corresponding to the block sections. Speed within block section is considered as a constant value and it changes when the corresponding signal is received from the next section. The micro-model is losing its effectiveness in the presence of random disturbances.

From the viewpoint of queuing theory all the points of conflict on open tracks and at stations may be interpreted as server systems. This is considered as the mesoscopic approach for the creation of train traffic model. The mesa-model assumes that an elementary checking element significantly exceeds the length of a block section. Checkpoints such as stations, stops and crossings are the element boundaries. The speed profile and a scheduled time interval between checkpoints here are taken as fixed values.

Random components, which are due to peculiar specific conditions of traffic and style of work of the driver, are reflected in a common distribution of the running time. In reality, coordinate control and centralized management of train runs (i.e. introduction of corrections) are made at the moments of train passage at the checkpoints. Decision to change the mode of run is made in the dispatch centre and is based on a mesa-model. Movement of a train along a spatial trajectory is represented as a set of elements between the control points. Complete time of a train passage by the trajectory is an additive value namely a sum of the elementary operation times. The trajectory of the train is modelled by a directed graph in the time domain (an activity graph), some nodes of which gather several arcs (Fig. 1).

The nodes of the graph represent the states of the process such as arrivals and departures on the station and the points of conflicts. Time deviation from the timetable is considered as an indicator of every state. The arcs of the graph reflect transition of the train from one state to another that is an activity operation. Some authors (e.g. Büker and Seybold [2]) include connections for 


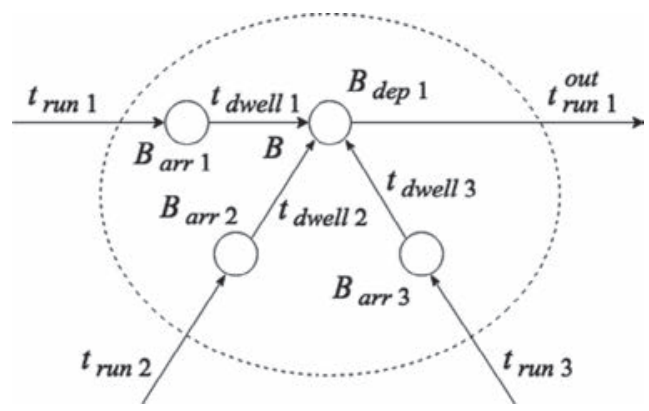

Figure 1: Fragment of the graph which reflects the passenger interlinking.

the passenger transfer and inter-train conflicts in this list. Length of the arcs reflects operating times that characterize the transition between checkpoints. Additional arcs correspond to delays that occur due to the influence of other trains, i.e. conflicts on sites or stations.

The approach considered is the basis for a generic stochastic model of train traffic on the railway section [13]. The process of accumulating the delays is represented as a tree of interferences, which is the graph whose nodes reflect the presence of conflicts between trains at the control points. It is assumed this points can be of two types, namely with primary (i.e. input) disturbances or with secondary delays. The arc length is considered as a random variable that distinguishes it from the most of previously reported frames.

\section{STOCHASTIC MODELLING OF CONTROL ACTIVITY}

The driver or the on-board system produces the permanent control over the motion parameters of the train. Speed is adjusted so as to perform the schedule. The time trajectory of the train is adjusted occasionally by the infrastructure manager. The purpose of regulation is timely arrival at checkpoints.

The running time is affected by random factors that are characterized by a probability density function. Over time, this function is changing, and its support becomes wider if there is no adjustment. At the same time adjustment by the system or the operator can stabilize the distribution and even to narrow its support.

Now we consider modelling the process of forming the probability distribution of deviation of the arrival time from the scheduled one, when there is a driver's control work. Let $t_{0}$ be the time of departure, and $s=s(t)$ and $v=v(t)$ traversed path and speed of the train, respectively. It is assumed that the minimum $v_{1}=v_{\text {min }}$ and maximum $v_{2}=v_{\max }$ allowable speeds are known. We denote by $v_{0}$ average speed of a train. Random factors lead to the scattering of trajectories (Fig. 2).

Let $\tau(s)$ be the actual arrival time of the train at $P$. If the train moves at a speed of $v_{j}$, the moment of its arrival is

$$
a_{j}(s)=t_{0}+\frac{s}{v_{j}}, j=0,1,2, \quad 0<\mathrm{s}_{1} \leq \mathrm{s} \leq \mathrm{s}_{2} .
$$

The random variable $Y(s)=\tau(s)-a_{0}(s)$ is the deviation from the scheduled arrival time $a_{0}(s)$. Note that $Y(s)$ takes the values of different signs.

Denote by $f(t ; s)$ the density function of the random variable $\tau(s)$, and by $g(t ; s)$ the density function of the random variable $Y(s)$. Obviously, 


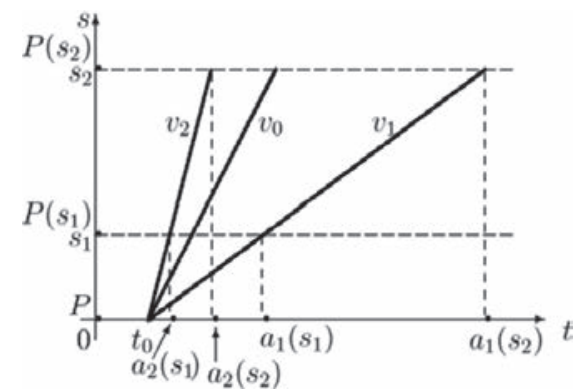

(a)

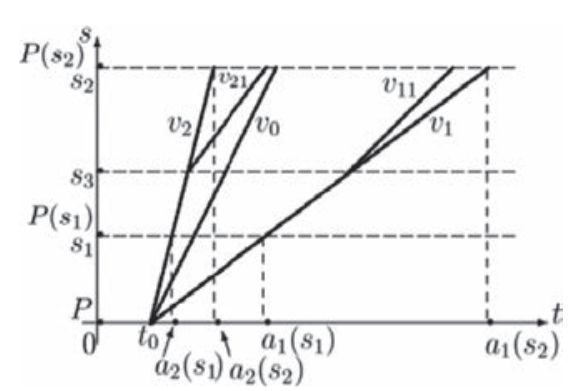

(b)

Figure 2: Graphs of functions $s=v_{j}\left(t-t_{0}\right), j=0,1,2$.

$$
g(t ; s)=f\left(t+a_{0}(s) ; s\right)
$$

The interval $\left[a_{2}(s), a_{1}(s)\right]$ is the support of $f(t ; s)$, i.e. $f(t ; s)=0$ when $t \notin\left[a_{2}(s), a_{1}(s)\right]$, $\left[a_{2}(s)-a_{0}(s), a_{1}(s)-a_{0}(s)\right]$ is the support of $g(t ; s)$.

Given the limitations on the speed, we obtain a one-to-one correspondence between the points of these intervals: $t^{\prime}=A\left(s_{2}\right) t+B\left(s_{2}\right)$, where $t \in\left[a_{2}\left(s_{1}\right), a_{1}\left(s_{1}\right)\right], t^{\prime} \in\left[a_{2}\left(s_{2}\right), a_{1}\left(s_{2}\right)\right]$,

$$
A(s)=\frac{a_{1}(s)-a_{2}(s)}{a_{1}\left(s_{1}\right)-a_{2}\left(s_{1}\right)}, \quad B(s)=\frac{a_{2}(s) a_{1}\left(s_{1}\right)-a_{2}\left(s_{1}\right) a_{1}(s)}{a_{1}\left(s_{1}\right)-a_{2}\left(s_{1}\right)},
$$

Note that the length of the interval $\left[a_{2}(s), a_{1}(s)\right]$ increases when the boundary condition on the permissible speed is not changed, i.e. if there is no intervention to regulate the movement. In this case, the scattering of the considered probability distribution increases. Speed change in some points, which produces the driver, results in a decrease of scattering.

Assumption. Suppose that for each fixed $s \tau(s) \stackrel{d}{=} A(s) \tau\left(s_{1}\right)+B(s)$, where $A(s) \neq 0$ and $B(s)$ are defined in eqn (3). This is equivalent to $Y(s) \stackrel{d}{=} A(s)\left(Y\left(s_{1}\right)+a_{0}\left(s_{1}\right)\right)+B(s)$. Here we have used the notation $W_{1} \stackrel{d}{=} W_{2}$ as random variables $\tau(s)$ and $[A+B]$ are identically distributed.

Note that if $f(t)$ is the density of the random variable $X, A \neq 0$ and $B$ are constants, then $\frac{1}{|A|} f\left(\frac{t-B}{A}\right)$ is the density of the random variable $A X+B$.

From this observation, assumption 1 and eqn (2), we deduce that

$$
\begin{gathered}
f(t ; s)=\frac{1}{|A(s)|} f\left(\frac{t-B(s)}{A(s)} ; s_{1}\right) \\
g(t ; s)=f\left(t+a_{0}(s) ; s\right)=\frac{1}{|A(s)|} g\left(\frac{t+a_{0}(s)-B(s)}{A(s)}-a_{0}\left(s_{1}\right) ; s_{1}\right) .
\end{gathered}
$$


Thus, we conclude that the density of the random variable $Y(s)$ with increasing $s$ is subjected to a linear transformation. With increasing $A(s)$ supports of densities $f(t ; s)$ and $g(t ; s)$ extend.

The intervention of the driver consists in change of speed at the point $s_{3}$. Speed is reduced, if the train is ahead of schedule, or increases in the opposite case (Fig. 2(b)). The distribution of the time of arrival becomes narrower.

The analysis shows that the limitations imposed, $v_{21}<v_{2}, v_{11}>v_{1}$, also result in linear transformation of $f\left(t ; s_{3}\right)$ density. The form of the distribution $g\left(t ; s_{2}\right)$ in the point $P$ is maintained, changing only its parameters. Experimental study on the Russian Railways shows that the real scattering of arrival times is well approximated by a gamma distribution. In the sections of railway, which carried out effective local control, the scattering width of the arrivals differs a little at different sites. The dispersion increases through the train route if the regulation is weak.

\section{ACTUALIZATION OF OPERATING TIME DISTRIBUTIONS}

In the real world, the actual train arrivals and failures of infrastructure in the coming hours are known quite accurately. This is a prerequisite to develop a more accurate prediction of traffic. The operational model is constructed by taking certain influencing factors into account. Thus, the specific parameters of operating times scattering can be deduced.

You must use the specific probability distribution of operating intervals to create a predictive model for each real situation. Such distributions for the running and stopping times are determined from historical data. By the historical data we mean the situations (scenarios) that occurred in previous periods on considered track section. Parameters of the operating time distributions depend on key factors like constraints on the train passage or weather conditions.

It is necessary to find out how to use historical data for a complete formalization of stochastic model of the train traffic. The idea of the early proposed method for determining the forecasted indicators on the basis of previous experience is known as situational heuristic predicting [16]. The method is based on constructing the array of experience $Z$, which is a set of indicators of the line functioning in certain real-life situation. This situation is determined by the values of influencing factors, such as the number of cars or trains at the adjacent stations, etc. The array $Z$ is a multidimensional matrix for various combinations of the factors and their associated cells that contain the realization times $\tau_{i}, 1 \leq i \leq n$, of a certain activity (e.g. travel time) in every historical situation. The array $Z$ is considered in the following general form:

$$
Z=\left(\begin{array}{cccc|c}
\Phi_{1}^{1} & \Phi_{2}^{1} & \cdots & \Phi_{m}^{1} & \tau_{1} \\
\Phi_{1}^{2} & \Phi_{2}^{2} & \cdots & \Phi_{m}^{2} & \tau_{2} \\
\vdots & \vdots & \ddots & \vdots & \vdots \\
\Phi_{1}^{n} & \Phi_{2}^{n} & \cdots & \Phi_{m}^{n} & \tau_{n}
\end{array}\right),
$$

where $\Phi_{j}^{i}$ denotes $i$ th particular observation of the influencing factor with number $j, 1 \leq j \leq m$ . Thus, sequence of values $\Phi_{1}^{r}, \Phi_{2}^{r}, \ldots, \Phi_{m}^{r}$ represents $r$ th historical situation with $r \in N$.

The paper [17] solves the problem of estimation of the process time $\tau_{n+1}$ pointwise. This assessment is carried out by means of the selection of situations, which are close to the $(n+1)$-th scenario. Note that two situations $i_{1}$ and $i_{2}$ are considered as close in case when 


\section{COMPRAIL}

the concrete realizations, $\Phi_{j}^{i_{1}}$ and $\Phi_{j}^{i_{2}}$, are similar in some predetermined way. The inequality $\left|\Phi_{j}^{i}-\Phi_{j}^{n+1}\right| \leq \varepsilon_{j}$ can be used as a criterion of closeness between the pair of situations, where $\varepsilon_{j}$ represents allowable deviation value of $j$ th factor. Seeking forecasted value $\tau_{n+1}$ is defined as the mean between $\tau_{k}$ from the close scenarios, i.e. by using the interpolation.

We propose a slightly different approach to the construction and use of the $Z$ array. The matrix includes process times that have been implemented for a certain set $N$ of previous actual situations. We assume the factors $\Phi_{j}$ are mutually independent random variables, and each of them has a distribution $F_{j}(x)=\mathrm{P}\left(\Phi_{j}<x\right)$. In contrast to the mentioned paper we do not compute a specific value $\tau_{n+1}$ but restore the distribution function of the random variable $\tau$. Method (a) of distribution restoring assumes the random variable $\tau$ considering the values $\tau_{1}, \ldots, \tau_{n}$ can be represented as a linear combination of all the factors, i.e.

$$
\tau=\sum_{j=1}^{m} \lambda_{j} \Phi_{j}
$$

where $\lambda_{j}$ are coefficients of sensitivity to changes in the factors $\Phi_{j}$.

We have the following system of equations:

$$
\left\{\begin{array}{c}
\Phi_{1}^{1} \lambda_{1}+\Phi_{2}^{1} \lambda_{2}+\ldots+\Phi_{m}^{1} \lambda_{m}=\tau_{1}, \\
\Phi_{1}^{2} \lambda_{1}+\Phi_{2}^{2} \lambda_{2}+\ldots+\Phi_{m}^{2} \lambda_{m}=\tau_{2}, \\
\ldots \ldots \ldots \ldots \ldots \ldots \\
\Phi_{1}^{n} \lambda_{1}+\Phi_{2}^{n} \lambda_{2}+\ldots+\Phi_{m}^{n} \lambda_{m}=\tau_{n} .
\end{array}\right.
$$

This system of equations is considered as overdetermined, because the number of equations $n$ exceeds the number of unknown variables $m$. Some 'optimal' vector solution $\lambda=\left(\lambda_{1}, \lambda_{2}, \ldots, \lambda_{m}\right)$ can be found by the method of least squares. The following relation is considered as a measure of closeness of the left and right sides of eqn (8):

$$
\sum_{i=1}^{n}\left(\tau_{i}-f_{i}(\lambda)\right)^{2} \rightarrow \min _{\lambda}
$$

where $f_{i}(\lambda)=\sum_{j=1}^{m} \Phi_{j}^{i} \lambda_{j}$. We calculate the required distribution after finding the vector $\lambda$ by using eqn (7). Determination of the density function of $\tau$ is reduced to ( $m-1)$-fold convolution of the densities of new random variables $\left(\lambda_{j} \Phi_{j}\right)$.

The density $f_{\tau}$ can be determined by the other approach (b). The method is based on the fact that an array of experience $Z$ contains a sample of values from the distribution of the random variable $\tau$. For this sample you can restore the law of distribution using the goodness of fit (e.g. Kolmogorov-Smirnov test or Chi-squared test). Thereby our approach is more common and informative than that in Ref. [16] and also allows the best use of the initial data $Z$.

Experimental studies provide the data that show the density of the arrival time to have a little asymmetric Gaussian. A closer approximation is a Weibull distribution. The main results are the following. The parameters of the arrival times density are almost identical for all the stations in the standard periods. But the dispersion increases an order of magnitude 
from 0.6 to $6.9 \mathrm{~min}^{2}$ at night (for the freight flow). At the same time the average delays are small. A similar pattern is in the suburban traffic.

\section{THE QUALITY MEASURE OF PASSENGER TRAIN TRAFFIC}

Punctuality in the passenger traffic segment is an important component of quality of the rail service. High level of punctuality increases the number of users of transport services and, consequently, income of the carrier and operator companies. Most studies on optimal traffic management have used the total cumulated delay as a measure of punctuality. However, this approach does not fully reflect the situation - how to completely satisfy the demands of the passengers.

The main characteristic of an operator which should be observed by him when he organizes the traffic control is as follows. The passenger begins to perceive the late arrival of the train as a significant delay only for sufficiently large values of delay. This measure has the uniqueness for every station and for different times of day. For example, a person perceives the delay more acutely during peak hours. Therefore, for the $i$ th situation in this period the threshold of perception $T_{d_{i j}}^{l i m}$ is smaller. The measure of quality of the service should not contain all the delays, but that lateness exceeds a specified threshold.

Another circumstance that must be considered is the damage caused by delays for different groups of passengers. Obviously, the approach is most appropriate when the priority service is given to numerous groups. One example is the traffic management on the suburban line. During rush hours, a manager devotes most attention to stations where there is mass boarding and alighting of passengers. He instinctively tries to make such adjustments to reduce the delays. Therefore, if the delay exceeds the threshold value, it is advisable to assess the damage by the number of passengers $M_{d_{i j}}$ who fell under the delay.

We formulate the following criterion for the quality of traffic in the heavy passenger train flow, using the approach just described. The scenario is considered the best, if its implementation gives delayed arrival of the minimum number of passengers at all the stations. In this case, the delays that exceed certain defined thresholds are taken into account:

$$
\sum M_{d_{i j}} \rightarrow \min , \tau_{i j} \geq T_{d_{i j}}^{l i m}, i=1,2, \ldots, m, j=1,2, \ldots, n,
$$

where the index ( $i j$ ) denotes the value of describing events occurring in the $i$ th interval at the time of day at the $j$ th station. Obviously the specified threshold must be peculiar for different types of trains such as high speed, intercity or commuter.

For example, the threshold value of delayed commuter trains during rush hours can be set from $1 \mathrm{~min}$ to $2 \mathrm{~min}$ in other periods. For those of local trains that have to deliver passengers on time at the connection station, this interval should be shortened to $0.5 \mathrm{~min}$. Using this rule it enables the separation of any delays into the groups, each of which obeys the following relation:

$$
\tau_{\mathrm{d}} \geq \tau_{\text {all } \mathrm{k}}
$$

In this case the objective function takes the following form:

$$
J(\Theta)=\sum_{k} n_{d e l \mathrm{k}}, \quad k=1, \ldots, K,
$$

where $K$ is the number of train groups with different boundary values $\tau_{a c \mathrm{k}}$. 


\section{CONCLUSIONS}

In this article, we present an approach that allows considering the effect of adjustments on the traffic characteristics. The model developed takes into account the actual situation and its impact on operating times. The probabilistic forecast uses historical data to determine the improved time distributions. The calculated results agree well with the real data on the train traffic. The proposed new criterion of punctuality provides more correct adjusting solutions.

The next step of this study is to use the model developed in solving a specific real-time problem. In particular, we will carry out a detailed study on a linear transformation of the arrival time distribution when the local adjustment is made.

\section{REFERENCES}

[1] Berger, A., Gebhardt, A., Müller-Hannemann, M. \& Ostrowski, M., Stochastic Delay Prediction in Large Train Networks. Proceedings of 11th Workshop on Algorithmic Approaches for Transportation Modelling, Optimization, and Systems (ATMOS'11), eds. A. Caprara \& S. Kontogiannis, pp. 100-111, 2011.

[2] Büker, T. \& Seybold, B., Stochastic modelling of delay propagation in large networks. Journal of Rail Transport Planning \& Management, 2, pp. 34-50, 2012. DOI: 10.1016/j. jrtpm.2012.10.001.

[3] Cacchiani, V., Huisman, D., Kidd, M., Kroon, L., Toth, P., Veelenturf, L. \& Wagenaar, J., Overview of recovery models and algorithms for real-time railway rescheduling. Transportation Research, Part B, 63, pp. 15-37, 2014. DOI: 10.1016/j.trb.2014.01.009.

[4] Törnquist, J., Computer-based decision support for railway traffic scheduling and dispatching: A review of models and algorithms. Proceedings of the 5th Workshop on Algorithmic Methods and Models for Optimization of Railways, (ATMOS'05), eds. L. Kroon \& H. Rolf, 23 p., 2005.

[5] Muhlhans, E., Berechnung der verspatungsentwicklung bei zugfahrten. Eisenbahntechn. Rundschau ETR, 39(7/8), pp. 465-468, 1990.

[6] Carey, M. \& Kwieciński, A., Stochastic approximation to the effects of headways on knock-on delays of trains. Transportation Research, Part B, 28, pp. 251-267, 1994. DOI: 10.1016/0191-2615(94)90001-9.

[7] Meester, L.E. \& Muns, S., Stochastic delay propagation in railway networks and phasetype distributions. Transportation Research, Part B, 41, pp. 218-230, 2007. DOI: 10.1016/j.trb.2006.02.007.

[8] Huisman, T. \& Boucherie, R.J., Running times on railway sections with heterogeneous train traffic. Transportation Research, Part B, 35, pp. 271-292, 2001. DOI: 10.1016/ S0191-2615(99)00051-X.

[9] Wendler, E., The scheduled waiting time on railway lines. Transportation Research, Part B, 41(2), pp. 148-158, 2007. DOI: 10.1016/j.trb.2006.02.009.

[10] Yuan, J., Stochastic Modelling of Train Delays and Delay Propagation in Stations, PhD Thesis, TRAIL Thesis Series no. T2006/6, the Netherlands, 142p., 2006.

[11] Kroon, L.G., Dekker, R. \& Vromans, M., Cyclic Railway Timetabling: A Stochastic Optimization Approach. Proceedings of the 4th Workshop on Algorithmic Methods and Models for Optimization of Railways (ATMOS'04), eds. F. Geraets, L. Kroon, A. Schoebel, D. Wagner \& C. Zaroliagiis, pp. 41-66, 2004.

[12] Vromans, M., Reliability of Railway Systems, PhD Thesis. TRAIL Thesis series no. T2005/7, The Netherlands TRAIL Research School, 244p., 2005. 
[13] Chebotarev, V., Davydov, B. \& Godyaev, A., Stochastic Traffic Models for the Adaptive Train Dispatching. Proceedings of the 1st International Scientific Conference on "Intelligent Information Technologies for Industry” (IITI'16), Rostov-on-Don, Russia, May 16-21, 2016.

[14] Goverde, R.P.M., A delay propagation algorithm for large-scale railway traffic networks. Transportation Research, Part C, 18(3), pp. 269-287, 2010. DOI: 10.1016/j. trc.2010.01.002

[15] Beck, A., Bente, H. \& Schilling, M., Railway Efficiency: An Overview and a Look at Opportunities for Improvement. The International Transport Forum, Discussion paper no. 2013-12, 44p., 2013.

[16] Shapkin, I.N., Yusipov, R.A. \& Kozhanov, E.M., Modelling train traffic based on multifactor setting technological operations. Vestnik VNIIZhT, 4, pp. 30-36, 2006.

[17] Törnquist Krasemann, J., Design of an effective algorithm for fast response to the rescheduling of railway traffic during disturbances. Transportation Research, Part $C$, 20(6), pp. 62-78, 2012. DOI: 10.1016/j.trc.2010.12.004. 